\title{
Sustain resource teachers in the appropriation of the graphic organizer to facilitate the understanding of disciplinary texts among at risk students at the secondary level
}

\author{
Nancy Granger, France Dubé1
}

\begin{abstract}
In Québec, the resource teacher role is assumed by secondary teachers without experience with at risk students or HDAA (MELS, 2006). Many of them say they have few ideas to sustain students or their teachers of different disciplines who deal with students with low literacy (Granger \& Dubé, 2014; Granger, 2012). Our research shows that support for resource teachers for reading and writing strategies can develop a metacognitive dialog by using the graphic organizer, a high cognitive recognized strategy to improve student understanding in all disciplines (Schoenbach, Greenleaf \& Murphy, 2012). This appropriation seems to better define the role of resource teachers both pedagogically than didactic.
\end{abstract}

Keywords: resource teacher, graphic organizer, at risk students, professionnal accompaniement, differentiated Instruction.

\begin{abstract}
Résumé
Au Québec, la fonction d'enseignant-ressource est assumée par des enseignants du secondaire n'ayant pas de formation particulière malgré qu'ils doivent intervenir auprès des élèves à risque ou HDAA (MELS, 2007). Plusieurs d'entre eux disent avoir peu de stratégies quant à la mise en œuvre d'un soutien utile à l'élève et aux enseignants des différentes disciplines du secondaire qui accueillent dans leur classe des élèves avec un faible niveau de littératie (Granger \& Dubé, 2014; Granger, 2012). Notre recherche montre qu'un soutien aux enseignants-ressources en matière de stratégies de lecture et d'écriture permet de développer un dialogue métacognitif par l'utilisation de l'organisateur graphique, une stratégie de haut niveau cognitif reconnue pour améliorer la compréhension des élèves dans toutes les disciplines (Schoenbach, Greenleaf \& Murphy, 2012). Cette appropriation semble permettre de mieux définir le rôle des enseignants-ressources tant au niveau pédagogique que didactique.
\end{abstract}

Mots-clés: enseignant-ressource, organisateur graphique, élèves à risque ou HDAA, accompagnement professionnel, différenciation pédagogique.

${ }^{1}$ Nancy Granger est Professeure associée Université de Sherbrooke; France Dubé est Professeure Université du Québec à Montréal. 


\section{Introduction}

$\mathrm{Au}$ Québec, la fonction d'enseignant-ressource existe depuis 2006 au secondaire. Elle a pour visée de soutenir les élèves à risque, handicapés, en difficulté d'adaptation ou d'apprentissage (HDAA). Pour la situer dans son contexte, cette fonction a été développée par le Ministère de l'Éducation, du Loisir et du Sport (MELS) à la suite de l'implantation de la nouvelle Politique de l'adaptation scolaire en 1999. L'intégration d'élèves à besoins particuliers nécessitait de fournir aux enseignants une ressource pour les aider à gérer cette nouvelle réalité. La fonction d'enseignant-ressource est attribuable à un enseignant à temps plein à qui l'on peut proposer jusqu'à 50\% de sa tâche d'enseignement (12 périodes sur 24) pour œuvrer auprès des EHDAA et offrir du soutien aux enseignants responsables d'enseigner différentes disciplines (français, mathématiques, histoire) (FSE, ENTENTE 2015-2020).

L'enseignant-ressource peut offrir un accompagnement personnalisé, notamment pour chaque élève qui entre au secondaire avec une année de retard; assumer un rôle de suivi scolaire et d'aide auprès d'élèves à risque, handicapé, en difficulté d'adaptation ou d'apprentissage (HDAA), particulièrement ceux ayant des difficultés relatives au comportement; assumer des tâches d'encadrement auprès de ces élèves et les soutenir, d'une part, dans leur démarche en vue de trouver des solutions à leurs problèmes et, d'autre part, dans diverses facettes de leur vie scolaire. L'enseignant-ressource travaille en concertation avec les enseignants responsables des élèves en difficulté qui lui sont adressés en portant une attention particulière aux enseignants en début de carrière. Enfin, il travaille en concertation avec les autres personnels de l'école (CPNCF, 2011, p. 188).

\section{Problématique}

Selon les encadrements légaux mentionnés ci-haut, la fonction d'enseignantressource ne nécessite pas de formation particulière pour intervenir auprès des élèves à besoins particuliers au secondaire. Les interventions attendues ont davantage à voir avec des rencontres à intervalles variables avec certains élèves qu'avec un soutien pédagogique suivi et structuré Or, nos travaux montrent que les enseignants-ressources disent avoir peu de stratégies ou d'idées quant à la mise en œuvre d'un soutien utile à l'élève et aux enseignants des différentes disciplines (Granger \& Dubé, 2014; Granger, 2012). Ils se disent également peu outillés pour intervenir dans ces différentes disciplines et souhaitent se rendre plus utiles auprès des élèves qui leurs sont référés. 
Peu de recherches se sont intéressées à la fonction d'enseignant-ressource à ce jour. Au Nouveau-Brunswick, Porter et Aucoin (2012), dans une recension exhaustive auprès des enseignants, montrent que les enseignants-ressources consacrent de 18 à 32,8\% de leur temps à effectuer d'autres tâches que le soutien aux enseignants ou aux élèves (Porter et Aucoin, p. 122). Les auteurs soulignent le peu de présence des enseignants-ressources en salle de classe et relèvent que des pratiques comme le modelage, le co-enseignement et les interventions en salle de classe auprès des élèves ayant des besoins particuliers sont encore peu exploitées par les enseignants-ressources (Ibid., p. 130). Au Québec, vérifier les devoirs non-faits, remplir l'agenda, donner des retenues, comptabiliser les absences ou encore faire le suivi motivationnel des élèves sont parmi les tâches qui nous ont été rapportées le plus souvent lors des recherches menées dans les milieux (Granger et Dubé, 2014).

Ainsi, la présente recherche s'articule sur la base de deux problématiques récurrentes et intimement liées au regard de la fonction d'enseignantressource. D'une part, les balises syndicales en vigueur encouragent peu le soutien didactique et pédagogique pouvant être apporté aux élèves ainsi qu'aux enseignants, ce qui a pour effet de reléguer les interventions de cette ressource au niveau des problématiques sociales (mentorat, tutorat) plutôt qu'au niveau de l'apprentissage. D'autre part, les enseignants-ressources se disent insatisfaits des rôles de soutien au comportement et de suivi organisationnel qui leurs sont attribués. Ils soulignent la nécessité d'intervenir sur l'apprentissage et de faire vivre des réussites scolaires aux élèves (Granger \& Tremblay, soumis). Or, lorsqu'il est question de mettre de l'avant des pratiques didactiques et psychopédagogiques différenciées favorables à l'apprentissage de l'ensemble des élèves, les enseignants et les enseignants-ressources disent être peu outillés et avoir peu de formation à cet égard (Allenbach, Borri-Anadon, Leblanc, Paré, Rebetez \& Tremblay, 2016). Au nombre des problématiques fréquemment identifiées lors des rencontres avec les enseignants-ressources au secondaire (Granger \& Dubé, 2014) et en général dans les écrits portant la réussite scolaire, les difficultés en compréhension de textes et le manque de vocabulaire spécifique sont souvent cités comme des obstacles à l'apprentissage pour les élèves tout venant, et particulièrement, pour les élèves à risque ou HDAA (Brochu, Deussing, Houme \& Chuy, 2013; CSE, 2013; NCES, 2009, MELS, 2008).

Les enseignants-ressources impliqués dans cette recherche souhaitaient être formés pour pouvoir intervenir d'un point de vue didactique sur l'apprentissage des élèves qui leur sont référés. Ils cherchaient des moyens pour engager les élèves dans les textes à lire dans les différentes disciplines et les soutenir pour qu'ils en dégagent le contenu essentiel du texte. En clair, il s'agissait de déterminer des moyens de remédiations en lecture-écriture qui seraient utiles à 
l'ensemble de la classe et plus spécifiquement, aux élèves ciblés. L'organisateur graphique s'est avéré le moyen choisi par les enseignants. La prochaine section traitera des recherches sur les interventions reconnues en lecture-écriture et présentera quels sont les avantages de l'utilisation de l'organisateur graphique dans un enseignement disciplinaire au secondaire.

\section{Cadre theorique}

Les difficultés en lecture-écriture éprouvées par les élèves du secondaire et l'impact de ces dernières sur la persévérance scolaire sont abondamment documentées (OECD, 2010). Selon le National Center for Education Statistics (NCES, 2009), plus de 60\% des élèves présenteraient des lacunes en lecture. Ils décrient la situation, mentionnant que ces difficultés sont d'importants freins à l'apprentissage que les élèves doivent faire dans les différentes disciplines et qu'elles risquent de nuire à leur réussite scolaire.

\section{Difficultés en lecture-écriture et les interventions reconnues}

Dans leur rapport, Lee, Grigg, et Donahue (2007) montrent qu'en lecture, les élèves de $8^{\mathrm{e}}$ année peinent à reconnaître l'information littérale dans le texte, à expliciter leur raisonnement, à faire des comparaisons, à trouver les idées principales et secondaires dans un texte, ou encore, à rapporter la trame de fond d'une histoire.

En écriture, plusieurs chercheurs (Graham \& Harris, 2009; Rouhani, Nafchi \& Ziaee, 2016) soulignent que les élèves en difficulté peinent, entre autres, à prendre des notes, à surligner et à composer des phrases explicatives. Ils ne savent pas comment catégoriser, structurer les informations en tenant compte des idées principales et secondaires. Lorsqu'ils doivent composer, la plupart de ces élèves cherchent à copier des passages directement du texte plutôt qu'à composer leurs propres phrases. Ces constats mènent les chercheurs à conclure que les interventions visant à soutenir l'appropriation de stratégies d'écriture et d'habiletés cognitives de haut niveau font encore peu partie des pratiques enseignantes. En ce sens, ils recommandent d'offrir du soutien et de l'accompagnement aux enseignants pour leur permettre d'intégrer dans leurs pratiques ces stratégies reconnues par la recherche.

Nous prenons également appui sur les résultats de recension et de métaanalyse pour soutenir le choix des interventions que nous avons privilégiées avec les enseignants-ressources. La méta-analyse de Scammacca, Roberts, Vaughn et Stuebing (2015) couvre la période de 1980 à 2011 chez des lecteurs 
qui éprouvent des difficultés entre la $4^{\mathrm{e}}$ et la $12^{\mathrm{e}}$ année, soit de la fin du primaire à la fin du secondaire. Parmi leurs objectifs de recherche, l'un d'eux consistait à connaître l'efficacité des interventions en lecture depuis les 30 dernières années. Leur étude met en valeur que des interventions comme l'étude de mots, la fluidité, le vocabulaire, la compréhension de même que des interventions, qui ciblaient différentes composantes en lecture à la fois, se sont avérées bénéfiques pour les élèves, qu'ils soient en difficulté ou non.

Dans une perspective plus disciplinaire, Kaldenderg, Watt et Therrien (2015) se sont intéressés aux difficultés en lecture en classe de sciences avec des élèves se situant entre la $5^{\mathrm{e}}$ et la $12^{\mathrm{e}}$ année. Ils ont analysé 12 articles, entre 1980 et 2012, portant sur les difficultés éprouvées par les élèves en lien avec la lecture en général, puis les interventions qui s'avèrent utiles pour l'apprentissage par les textes informatifs utilisés en sciences. Ces auteurs s'intéressent particulièrement aux élèves ayant un trouble d'apprentissage intégrés en classe ordinaire et qui présentent des lacunes en lecture. Parmi leurs questions de recherche, l'une d'elles visait à savoir quelles sont les interventions les plus efficaces pour améliorer la compréhension en lecture de textes en classe de sciences? Leur analyse montre que pour accroître la compréhension en lecture en classe de sciences, l'enseignement du vocabulaire doit se faire en concomitance avec l'organisation graphique. L'utilisation de l'enseignement direct et le soutien des élèves avec des stratégies mnémotechniques seraient aussi pertinents, surtout s'ils sont combinés avec des stratégies métacognitives qui valorisent les stratégies de haut niveau comme se questionner sur les relations de causalité et sur les inférences. Kaldenberg et al. (2015), soutiennent que lorsque les habiletés de base ne sont pas maitrisées, ces élèves performent sous la moyenne et continuent à prendre du retard par rapport aux autres élèves. Selon ces chercheurs, il serait pertinent que les enseignants des différentes disciplines comme les sciences, les mathématiques ou l'univers social soutiennent ces élèves en difficulté en lecture afin qu'ils s'approprient le contenu disciplinaire et qu'ils puissent tisser des liens entre les stratégies en lecture et les contenus disciplinaires.

Dans le même esprit, McKenna, Shin et Ciullo (2015), ont réalisé une synthèse portant sur la compréhension en lecture chez les élèves en difficulté dans les cours de mathématiques. Ils cherchaient à dresser le portrait d'interventions reconnues comme efficaces (evidence-based practices) recensées entre 2000 et 2013. Ils concluent, entre autres, que peu de soutien en compréhension de texte est offert aux élèves, et que les enseignants appliquent les stratégies de lecture de façon plus ou moins adéquate. De plus, un écart important existerait entre les recommandations en vigueur dans les recherches et les pratiques d'intervention observées dans ces milieux. Ils relèvent une faible occurrence de l'enseignement de stratégies de compréhension au 
secondaire et recommandent que des recherches futures étudient l'utilisation de stratégies pédagogiques efficaces au sein du curriculum que ce soit en mathématiques ou dans d'autres disciplines, tant au primaire qu'au secondaire.

Enfin, la synthèse de Graham et Harris (2009) sur l'écriture propose un regard sur 30 ans de recherches consacrées au développement de stratégies d'enseignement pour soutenir les élèves en difficulté ou ceux ayant un trouble d'apprentissage. Ces travaux ont mené à développer le modèle selfregulated strategy development (SRSD) basé sur la planification, la révision et l'autorégulation du processus d'écriture dans le but de répondre aux besoins éprouvés par les enseignants. Ces chercheurs visaient à composer des textes avec des élèves qui éprouvaient de la difficulté à organiser leur travail, qui possédaient des connaissances de base en écriture lacunaires ou absentes et qui manifestaient peu de motivation à la tâche. Durant ces années, ils ont fait des revues de littérature portant sur les pratiques d'intervention efficaces en écriture pour les élèves en difficulté ou présentant un trouble d'apprentissage (Graham et Perin, 2007). Parmi les conclusions, ils soulignent que a) plus les élèves ont de hauts niveaux d'habiletés en écriture, plus ils performent; b) développer des scripteurs habiles nécessite l'enseignement de stratégies plus complexes lorsque le niveau scolaire augmente; c) la capacité des élèves à utiliser les stratégies enseignées est un bon indicateur des performances en écriture; d) et, enfin, l'enseignement de stratégies de rédaction permet aux élèves de s'améliorer significativement.

Toutefois, Graham et Harris (2009) soulèvent quelques défis relatifs à l'enseignement de l'écriture. Ces derniers ont observé que près de $40 \%$ des enseignants n'enseignent pas de stratégies d'écriture de textes et ne soutiennent pas les élèves ayant des difficultés en rédaction. Les chercheurs ont noté également que l'enseignement de certaines stratégies est inadéquat et doit faire l'objet de perfectionnement chez ce personnel. D'ailleurs, ils ont documenté que plusieurs enseignants se sentent peu préparés pour enseigner ces stratégies.

$\mathrm{Au}$ Canada, Brochu, Deussing, Houme et Chuy (2013) portent à notre attention une diminution significative des niveaux d'habiletés en lecture des jeunes canadiens. Ils préconisent un rehaussement des ressources pédagogiques nécessaires (moyens, méthodes, stratégies) afin de mieux préparer les élèves à relever les défis de notre société. Les chiffres avancés par McCraken \& Murray (2009) montrent que 20 à 40\% des élèves canadiens n'ont pas les compétences suffisantes en lecture-écriture pour se débrouiller au quotidien. Alors qu'au Québec, les enquêtes montrent que ce serait $40 \%$ des jeunes entre 10 et 15 ans qui n'ont pas la préparation nécessaire pour lire, écrire et communiquer efficacement au quotidien. À cet égard, le ministère de l'Éducation, des Loisirs et du Sport (MELS, 2008) suggère d'accroître le niveau de préparation des enseignants et de renforcer les mesures de soutien en lecture et en écriture. 
Ainsi, former les enseignants-ressources à utiliser les stratégies de lectureécriture reconnues par la recherche comme étant favorables à la réussite des élèves apparaît une solution intéressante pour rehausser la valeur pédagogique de leur accompagnement. Sachant que tous les enseignants-ressources sont d'abord des enseignants dans diverses disciplines (histoire, sciences, langue), cette formation pourra être utilisée dans une double perspective soit de a) soutenir les élèves à risque ou HDAA qui leur sont confiés (fonction d'enseignant-ressource), et b) intégrer ces stratégies au sein de leur enseignement (fonction d'enseignant disciplinaire).

\section{Apport des organisateurs graphiques à la compréhension de textes}

Dexter et Hugues (2011) se sont intéressés à l'apport des organisateurs graphiques dans la réussite des élèves ayant des difficultés d'apprentissage. Leur méta-analyse porte sur des recherches qui incluent des élèves de la fin du primaire et du secondaire. Leur choix s'est porté sur cette catégorie d'âge parce cela correspond au moment où le curriculum se complexifie et que la compréhension de textes nécessite des compétences didactiques spécifiques pour aborder les textes disciplinaires qui sont proposés à l'école. Parmi les résultats recensés, ils relèvent les effets positifs d'un enseignement explicite de l'organisateur graphique. L'aspect visuel permettrait un meilleur rappel des connaissances et son utilisation permettrait aussi la mémorisation à plus long terme des éléments de contenu. Les auteurs suggèrent notamment aux enseignants de débuter à l'aide de cartes sémantiques puis de poursuivre à l'aide de schémas pour réviser les notions et tisser des liens entre les éléments vus en classe. Parmi les avantages, ils relèvent l'identification des idées principales et secondaires ainsi que la construction de sens induite par le modelage et les discussions métacognitives suscitées par l'enseignant. Parmi les défis à relever, Dexter et Hughes mentionnent la production de modèles d'utilisation de l'organisateur graphique éprouvés par la recherche.

Les avantages relevés dans cette méta-analyse sont aussi soutenus par d'autres chercheurs. Chaque fois, le rôle de l'enseignant dans l'utilisation de cet outil apparaît indéniable. Citons à cet égard Schoenbach, Greenleaf et Murphy (2012) qui décrivent l'organisateur graphique comme un excellent moyen pour conduire des discussions métacognitives en salle de classe. Il permet d'activer les connaissances antérieures, de mobiliser les informations données dans le texte, de se questionner et de construire du sens en fonction d'une intention de lecture spécifique, et ce, dans plusieurs disciplines scolaires. Selon Lee et Spartley (2010), l'enseignant joue un rôle majeur lorsqu'il s'agit de rendre accessible le contenu des textes disciplinaires. Block et Pressley 
(2007) proposent que les enseignants procèdent par étapes pour faciliter la compréhension d'un texte. D'abord, ils doivent s'assurer que les élèves comprennent les mots, les phrases ou les paragraphes, ensuite les textes, puis construire les apprentissages et les utiliser. Pour y arriver, les enseignants doivent avoir une bonne connaissance des processus cognitifs en jeu et des moyens qui facilitent l'explicitation, à chacune des étapes.

Les enseignants peuvent l'utiliser pour tisser des liens entre des concepts ou pour recréer une séquence de type cause/conséquence en vue d'expliquer un phénomène (Strangman, Hall \& Meyer, 2003). Selon ces derniers, différentes représentations graphiques peuvent être utilisées en fonction de l'intention pédagogique. L'arborescence permet de hiérarchiser des idées ou des concepts. Le diagramme de Venn soutient la comparaison de phénomènes en sciences ou en univers social, il peut aussi permettre de comparer des lieux ou des personnages dans des textes littéraires. Le tableau à double ou plusieurs entrées aide à classifier des informations ou à mettre en relation des données obtenues. La carte sémantique, quant à elle, permet de mettre en relation des mots de vocabulaire qui se rapportent à un même thème ou à une même idée. Enfin, la carte conceptuelle mettra en relation différents concepts et stimulera la vue d'ensemble d'un module ou d'une thématique. Dans tous les cas, Chauvin et Theodore (2015) recommandent aux enseignants de se familiariser avec l'outil de manière à pouvoir engager les élèves dans des discussions métacognitives autour des informations choisies. Cette appropriation apparaît nécessaire puisque plusieurs auteurs questionnent l'efficacité de cet outil lorsque les enseignants ne sont pas pleinement à l'aise avec son utilisation. Parmi les dérives répertoriées, le peu de soutien en cours de réalisation de l'organisateur graphique et une utilisation dénuée de sens relègueraient l'activité au simple remplissage d'un tableau ou d'un schéma sans y associer des questionnements de haut niveau cognitif.

Comme mentionné dans la problématique, puisque le rôle des enseignantsressources est actuellement peu précisé, ces derniers se rabattent sur des tâches davantage administratives (gestion de l'agenda de l'élève, méthode d'organisation des devoirs et travaux à remettre) plutôt que pédagogiques. Dans une perspective où l'on s'attend de la part des enseignants-ressources qu'ils fassent preuve de différenciation pédagogique, qu'ils élaborent des moyens pour réguler les apprentissages des élèves et qu'ils collaborent avec leurs collègues enseignants, l'organisateur graphique nous est apparût comme un outil fédérateur pour l'engagement de tous. À cet égard, nous avons voulu développer la compétence des enseignants-ressources à les utiliser dans leurs accompagnement pédagogique auprès des élèves à risque et en difficulté et sonder la pertinence d'intégrer cet outil pour soutenir leurs interventions 
auprès des élèves et des enseignants au regard de la compréhension des textes disciplinaires au secondaire.

Les objectifs spécifiques de cette étude exploratoire avaient pour but de:

1. Décrire les pratiques pédagogiques qui découlent de l'utilisation de différents types d'organisateurs graphiques.

2. Décrire les apprentissages perçus par les élèves en difficulté lors de l'utilisation de différents types d'organisateurs graphiques.

3. Réfléchir sur l'intérêt d'intégrer un outil didactique tel que l'organisateur graphique pour enrichir l'accompagnement pédagogique offert par l'enseignant-ressource au secondaire.

La partie qui suit présente la méthodologie utilisée dans cette recherche au regard des buts visés par les participants.

\section{Methodologie}

Notre projet s'inscrit dans le cadre d'une recherche-action-formation au sens convenu par Karsenti et Savoie-Zajc (2004, p. 186), soit de susciter un changement dans le but d'améliorer une situation donnée, de favoriser la compréhension des pratiques, de permettre l'évaluation, la résolution de problèmes ou encore, la production de connaissances. Pour y arriver, Prud'homme, Dolbec et Guay (2011) suggèrent de valoriser les interactions, la réflexion sur une problématique, de soutenir la formulation d'un besoin, la planification des actions à poser, le passage à l'action et l'évaluation de l'expérimentation par l'équipe-école. La démarche de recherche-action s'inscrit dans un processus synergique qui allie la recherche à la pratique (Couture, Bednarz \& Barry, 2007).

Afin que les enseignants-ressources puissent bénéficier pleinement du caractère itératif de ce type de démarche, nous leur avons proposé de participer à un dispositif d'accompagnement à deux volets développés lors de notre recherche doctorale (Granger, 2012). Le premier volet consistait à offrir aux enseignants-ressources des formations théoriques en groupe portant sur les caractéristiques des élèves en difficulté, les stratégies d'apprentissage en lecture et en écriture dont l'utilisation des organisateurs graphiques pour mieux comprendre les textes lus (Schoenbach, Greenleaf \& Murphy, 2010). Les types de questionnements à adresser aux élèves ont été d'abord abordés pour structurer les interactions des enseignants avec les élèves (Bissonnette, Richard, Gauthier \& Bouchard, 2010). Le deuxième volet consistait à leur offrir un soutien individualisé, ou en sous-groupe, afin qu'ils réalisent des organisateurs graphiques en lien avec le contenu disciplinaire. Lors de ces moments d'accompagnement, la chercheuse-formatrice rendait explicite toutes les 
considérations théoriques et pratiques qu'elle prenait en compte pour élaborer un organisateur graphique afin que l'enseignant-ressource puisse utiliser cette démarche comme modèle par la suite. Cette étape de coconstruction des savoirs engendrait un questionnement réciproque entre la chercheuse-formatrice et l'enseignant-ressource et permettait de clarifier le rôle de ces derniers en fonction des besoins des élèves et des enseignants avec lesquels ils doivent collaborer (Bourrassa, Philion \& Chevalier, 2007).

\section{Participants}

Notre recherche s'est déroulée entre 2012 et 2014 dans une commission scolaire de la rive-nord de Montréal, au Québec. Un total de neuf enseignantsressources ont manifesté leur l'intérêt à apprendre à enseigner la stratégie de l'organisateur graphique à leurs élèves à risque et en difficulté. Ils détenaient entre 5 et 22 années d'expérience, étaient enseignants de français $(\mathrm{n}=2)$, univers social $(n=3)$, mathématiques $(n=3)$, sciences $(n=1)$ et avaient été choisis pour occuper la fonction d'enseignant-ressource.

Nous avons demandé aux élèves qui avaient reçu du soutien des enseignantsressources s'ils voulaient participer à un groupe de discussion. Cinq élèves de la quatrième secondaire et cinq élèves de première secondaire provenant de deux écoles différentes (école $1=1^{\text {re }}$ secondaire; école $2=4^{\text {e }}$ secondaire) ont accepté de participer aux groupes de discussions. Nous avons rencontré les groupes séparément durant une période de classe. Les parents, les enseignants et la direction de chaque école ont signé une feuille de consentement éthique.

\section{Collecte de données}

Mentionnons que la formation dont il est question s'est inscrite dans le cadre d'une recherche-action-formation plus large qui comptait en tout 29 enseignants-ressources. Cette dernière était déjà encadrée par une démarche réflexive en partenariat qui comportait deux rencontres annuelles en groupe de discussion afin de clarifier les besoins et les attentes des enseignants-ressources (Boudreault \& Kalubi, 2006). Pour le segment spécifique de la formation portant sur les organisateurs graphiques, une rencontre de départ a eu lieu avec les neuf enseignants-ressources intéressés et une entrevue semi-dirigée a été réalisée individuellement à la fin de chaque année. L'objectif de cet article n'est pas de décrire à proprement dit la démarche de formation. Il s'agit plutôt de relever les effets perçus par les enseignants sur la compréhension de texte dans les disciplines visées et la réussite des élèves en difficulté. 
Les données ont été collectées par le biais d'un groupe de discussion en début d'année et par une entrevue semi-dirigée réalisée à la fin de l'année. Lors du groupe de discussion, les participants ont relevé les difficultés qu'ils éprouvaient à aider véritablement les élèves, ont précisé leurs attentes et au regard des différentes propositions qui ont émergées et ont choisi d'expérimenter l'utilisation de l'organisateur graphique. À la fin de l'année, les participants ont été interrogés sur l'apport de l'organisateur graphique dans l'apprentissage des élèves. Nous leur avons demandé de s'exprimer sur les facilitateurs perçus et les obstacles rencontrés. Nous les avons également invités à décrire leur rôle d'enseignant-ressource à travers l'utilisation de cet outil.

Dans le but de connaître les perceptions des élèves qui avaient vécu l'utilisation de l'organisateur graphique en classe ou en sous-groupe de besoins, nous avons tenu deux groupes de discussion avec des élèves volontaires. Le premier était composé de cinq garçons de quatrième secondaire en mathématiques et le second de trois garçons et deux filles de première secondaire en français et en univers social. Nous les avons interrogés sur l'apport de l'organisateur graphique dans leur compréhension des textes lus dans les disciplines et les effets sur leur réussite scolaire.

Tout au long du processus, la chercheuse-formatrice a complété un journal de bord où elle consignait des observations telles que: le contenu abordé, les besoins manifestés par les élèves ou par les enseignants-ressources, les modalités mises en place pour soutenir les enseignants-ressources et les stratégies abordées lors des formations qui ont ensuite été transférées en salle de classe. Ces écrits ont contribué à documenter les rencontres qui ont eu lieu en cours d'année pour soutenir les enseignants-ressources dans leur appropriation de l'organisateur graphique (Mertens, 2005).

\section{Analyse des données}

Les énoncés recueillis dans les groupes de discussions et au cours des entrevues semi-dirigées ont été transcrites puis analysées à l'aide du logiciel QDA miner (Lewis \& Maas, 2007). Une grille de codification a permis de créer des catégories portant sur les pratiques pédagogiques visant une amélioration de la compréhension des textes lus notamment au regard de l'utilisation des organisateurs graphiques par les enseignants-ressources dans le soutien apporté aux élèves et aux enseignants. Le point de vue des élèves a été codé séparément et a permis de nuancer les énoncés recueillis chez les enseignants-ressources.

La prochaine section décrit, en premier lieu, les nouvelles pratiques pédagogiques, telles que perçues par les enseignants-ressources qui ont utilisé 
l'organisateur graphique pour soutenir la compréhension des élèves dans la lecture de textes disciplinaires au secondaire. En second lieu, les apprentissages perçus par les élèves seront décrits au regard des facilitateurs et des obstacles à la compréhension lorsqu'ils abordent une tâche disciplinaire. Enfin, les propos recueillis auprès des enseignants-ressources concernant l'intérêt d'intégrer un outil didactique tel que l'organisateur graphique pour enrichir le rôle que peut avoir l'enseignant-ressource au secondaire seront rapportés.

\section{Resultats}

Cette recherche visait à accompagner les enseignants-ressources à développer de nouvelles stratégies de soutien. Ils souhaitaient explorer un outil pour soutenir l'apprentissage des élèves à besoins particuliers et pour mieux accompagner les enseignants avec qui ils collaborent au quotidien. Ainsi, les objectifs poursuivis étaient de 1) décrire les pratiques pédagogiques qui découlent de l'utilisation de différents types d'organisateurs graphiques; 2) décrire les apprentissages perçus par les élèves en difficulté lors de l'utilisation de différents types d'organisateurs graphiques, et 3) réfléchir sur l'intérêt d'intégrer un outil didactique tel que l'organisateur graphique pour enrichir l'accompagnement pédagogique offert par l'enseignant-ressource au secondaire. Ce faisant, ils voulaient également développer l'autonomie des élèves dans leur façon d'aborder les textes à lire. Les cours de mathématiques et de sciences de quatrième secondaire ainsi que de français et d'univers social de première secondaire ont été des lieux d'expérimentation de l'organisateur graphique par les enseignants-ressources.

\section{Les pratiques pédagogiques}

Les enseignants-ressources sont généralement utilisés pour assurer un suivi scolaire portant sur les comportements, habitudes ou méthodes de travail des élèves. Les résultats présentés dans cette section montrent qu'ils peuvent être fort utiles pour l'enseignement de stratégies de compréhension qui engagent véritablement les élèves dans leur apprentissage et les amène à établir des liens entre les notions lues.

a) L'organisateur graphique comme soutient à l'apprentissage

Les enseignants-ressources relèvent la qualité des interactions possibles avec les élèves quant au contenu abordé: "Je vois une différence sur leur capacité à discuter de la matière, ils font plus de liens». Ces amélioration perçues leur 
font prendre conscience de l'impact de leurs interventions: «Avant, j'arrivais trop vite avec des solutions, maintenant, je me permets plus de les questionner, je permets davantage aux élèves en difficulté de faire un bout de chemin» (ER, univers social et mathématiques). Ils sont aussi capables de verbaliser leurs intentions pédagogiques et de justifier l'utilisation de l'outil: «Souvent à force de les faire verbaliser, ils intériorisent ce qu'ils doivent faire; les organisateurs graphiques ont beaucoup servi à comparer, à concevoir la matière et à étudier» (ER, univers social, français, sciences et mathématiques); «Je les ai utilisés pour structurer les idées, faire des résumés. Les élèves les sortaient et s'y référaient; j'aurais aimé connaître cet outil quand j'étais élève!; je me sens à l'aise de le réutiliser, ça résume bien et c'est visuel» (ER, univers social, français).

b) L'organisateur graphique comme outils de révision

Les enseignants-ressources rappellent combien la préparation à l'examen de mathématiques était laissé aux soins des élèves: Avant, «les élèves avaient le droit à une feuille de notes recto-verso, je leur ai dit qu'ils devaient être autonomes et la faire seule. Ils sont arrivés à l'examen avec une feuille toute croche, ils étaient fébriles, désorganisés, pas solides». Dans le cadre du projet, «On a décidé de travailler mieux la révision et d'accompagner la structuration de la feuille de note. Pour chaque cours de révision on a fait des tableaux synthèses. Ça, c'est beaucoup de travail!»; «Comme enseignante-ressource j'ai vu que les élèves étaient beaucoup plus calmes et plus organisés, cela veut dire qu'il y a eu une belle amélioration» (ER, mathématiques).

c) L'organisateur graphique comme levier pour une autonomie accrue chez les élèves

Les interventions structurées des enseignants-ressources semblent avoir permis aux élèves de s'approprier une démarche qu'ils ont pu reproduire: Avant, «Quand la prof finissait d'expliquer, elle donnait des problèmes à faire individuellement. Après deux minutes, les mains se levaient et les élèves disaient qu'ils ne comprenaient pas. Comme ER je passais mon temps à leur répéter la même chose. Lorsqu'on a initié les organisateurs graphiques sous forme de tableaux, je me suis rendue compte qu'ils s'appropriaient davantage ce qu'ils doivent faire»; «En construisant le tableau pour eux, j'ai dû me questionner: Qu'est-ce qui est important? Quel est le sujet? Je voudrais continuer à y travailler et à les améliorer»; «Cette démarche les a beaucoup sécurisés, ils sont maintenant capables d'en faire» (ER, univers social, $2^{\mathrm{e}}$ et $4^{\mathrm{e}}$ secondaire). 
d) L'organisateur graphique comme synthèse d'informations pour structurer sa pensée

Au départ, le manque de stratégies des élèves était décrié: «En français, la plupart ne veulent même pas lire un texte. Ils savent qu'ils ne retiendront rien»; «En univers social, il y a beaucoup de choses à retenir. Si je ne structure pas leur feuille de notes, ils écrivent n'importe comment les informations relevées dans les textes. Ils pensent que tous les cours sont différents, ils ne font pas de liens. Aussi, lire des cartes, interpréter un territoire en géographie, ils ont de la difficulté, il y a trop d'informations pour eux, ils passent par-dessus des schémas et des tableaux qui sont importants. Des fois, je suis découragée». Une fois que ces enseignants ont structuré le contenu à apprendre et fourni des organisateurs graphiques pour consigner les idées principales et secondaires, ils ont mentionné: «C'est vraiment utile dans la façon d'aborder le texte». Les élèves ne le voient plus comme un paquet de pages à lire l'une en arrière de l'autre. Il y a des titres et des intertitres qui me servent à comprendre. Ils peuvent faire des regroupements d'idées et aller les déposer en quelque part (dans l'organisateur). Cela permet de finir avec une seule feuille qui résume ce qu'il y avait d'important dans le texte» (ER, français, univers social).

Pour le texte courant: «On a modélisé comment on s'y prend pour choisir ce qui est important. Soit, ils soulignent tout ou, ils ne soulignent rien. L'organisateur a permis de reformuler étant donné que l'organisateur contenait plus des courtes idées ou des mots-clés. L'organisateur leur a aussi permis de faire des regroupements et donc de résumer» (ER, français $1^{\text {re }}$ secondaire).

Les enseignants-ressources ont montré un bel enthousiasme quant à leur appropriation des différents types d'organisateurs graphiques expérimentés avec les élèves et les enseignants, ils se sont nourris des succès vécus par plusieurs élèves. Bien entendu, l'appropriation des stratégies était inégale selon les enseignants-ressources mais elle faisait partie de leurs préoccupations et constituait une occasion de formation continue sur un rôle encore inexploité dans le cadre de cette fonction.

\section{Apports des organisateurs graphiques sur l'apprentissage selon les élèves}

Nous avons réalisé des entretiens avec les élèves qui ont bénéficié de l'enseignement-ressource en quatrième secondaire afin de recueillir leur perception des effets sur leurs apprentissages.

a) Sur le développement de stratégies pour comprendre

Les élèves témoignent de leurs nouvelles habiletés et des effets perçus sur leur réussite: «J'ai appris à faire des feuilles de notes et je suis moins stressé 
aux examens»; «Les organisateurs graphiques nous évitent de chercher dans notre livre; les théorèmes sont là, on voit comment la matière est organisée»; «Avant, ma stratégie était d'apprendre par cœur, ça ne marchait pas parce que rendu à l'examen, j'avais une perte de mémoire. Maintenant, je sais que l'organisateur graphique m'aide à me souvenir et mon stress a diminué» (Élèves de $4^{\mathrm{e}}$ secondaire).

L'aspect visuel semble avoir aidé à classer et à interpréter l'information: «On a fait des affiches (organisateurs graphiques) sur des notions utiles. Ça aide vraiment beaucoup»; «On partait de deux cercles (diagramme de Venn) avec un texte, puis, il fallait trouver les ressemblances et les différences. Ça aidait à écrire et à apprendre sur le sujet» (Élève de $1^{\text {re }}$ secondaire).

b) Sur la capacité à réinvestir ce qu'on a appris sur le sujet

Lors de productions écrites en français, les organisateurs graphiques ont été utilisés comme plan et ont aidé à colliger des informations qu'ils ont été réinvesties dans leur texte: «Ça aide à faire des productions écrites, à savoir des choses sur le sujet».

En mathématiques, les élèves de $4 \mathrm{e}$ secondaire vivaient beaucoup de surcharge cognitive et étaient tentés de ne plus faire la tâche. Les organisateurs graphiques ont encouragé les élèves à persévérer: «On a de la difficulté à faire une synthèse dans notre tête. On vient mêlés. Les schémas aident vraiment beaucoup».

Ces entretiens, réalisés avec les élèves qui ont été accompagnés par les enseignants-ressources, ont mis en lumière une grande détermination à réussir à l'école. Ce qui était très frappant, c'est l'empressement avec lequel ils ont accueilli l'aide des enseignants-ressources en classe et les efforts qu'ils ont fait pour réussir. Cet enthousiasme, de part et d'autre, a contribué à alimenter notre réflexion quant au rôle de l'enseignant-ressource au secondaire ainsi qu'aux moyens à mettre en place pour soutenir à la fois les enseignants disciplinaires et les élèves à risque ou HDAA. Dans cette étude, les élèves ciblés étaient en très grande difficulté et avaient connu un parcours scolaires difficile. Il semble que cette trajectoire scolaire n'ait pas entravé leur capacité à mieux comprendre des textes disciplinaires en développant des stratégies favorables à une meilleure compréhension. 
Intérêt de l'organisateur graphique dans les rôles et fonctions de l'enseignant-ressource

Les enseignants-ressources souhaitaient développer de nouvelles pratiques dans le cadre de leur fonction. Voici quelques propositions à l'issue du projet: «L'ER peut davantage jouer le rôle d'activateur de connaissances et de facilitateur à l'apprentissage puisqu'il responsabilise les élèves, il discute avec eux et les renvoie aux stratégies apprises et aux outils pédagogiques mis à leur disposition». Différentes pratiques pédagogiques sont d'ailleurs identifiées comme nouvelles: «J'ai monté beaucoup de matériel, je me soucie à la fois des élèves et de l'enseignant, je veux pouvoir être utile aux deux; des fois, je lève la main et je pose des questions pour amener l'enseignant à mieux expliciter sa pensée aux élèves; ce que j'ai compris en faisant de l'enseignement-ressource c'est qu'il y a des stratégies et des façons de faire qui se retrouvent dans toutes les matières. C'est cet aspect-là que l'ER doit apporter. À titre d'exemples les ER mentionnent comment ils fonctionnent pour aborder les difficultés d'apprentissage: «Maintenant, je mets de l'importance à identifier l'intention pédagogique: Qu'est-ce que je veux que les élèves sachent ou puissent faire? Aussi, je me demande: les textes sont-ils accessibles pour les élèves? Le vocabulaire est-il trop difficile? Dois-je utiliser une autre formulation?». Un enseignant-ressource qui est aussi intervenu dans deux disciplines a perçu la pertinence des stratégies générales en lecture: «Cette année, j’ai pu travailler deux matières (sciences et français) en utilisant des stratégies similaires soit les stratégies de lecture et les organisateurs graphiques. Pour moi, c'est clair que c'est aidant. Les organisateurs ont révolutionné ma façon d'aborder les textes».

Des défis restent toutefois à relever: «J'aimerais bâtir de nouvelles choses; j'avoue que des idées nouvelles, j'en ai pas vraiment»; «J'ai de la misère à être proactive, je ne me trouve pas toujours optimale». Ils souhaitent d'ailleurs continuer à développer leurs habiletés didactiques pour pouvoir disent-ils «mieux travailler le contenu essentiel avec les élèves en difficulté».

\section{Discussion}

La fonction d'enseignant-ressource a été développée dans le but de soutenir l'intégration et la réussite scolaire des élèves à risque ou HDAA en classe ordinaire au secondaire. Dix années se sont écoulées depuis la mise en place de cette fonction sans qu'à ce jour des balises claires ne structurent l'agir professionnel des enseignants à qui l'on confie cette importante mission. Les encadrements légaux (FSE, 2015-2020; CPNCF, 2011) fournissent bien entendu un point de départ mais demeurent encore flous. Au terme de deux recherches- 
actions qui cumulent maintenant huit années d'accompagnement d'enseignantsressources, nos rencontres sur le terrain nous forcent à constater le besoin qu'ont ces enseignants de s'impliquer auprès des élèves dans ce qu'ils savent le mieux faire, enseigner (Granger, 2012; Granger et Dubé, 2014). Les tâches administratives ou les rencontres motivationnelles ne trouvent pas véritablement d'issues si les élèves ne vivent pas de réussites. Nous sommes conscientes du caractère exploratoire de ce projet, mais il a permis d'ouvrir une fenêtre sur un volet plus pédagogique de la fonction d'enseignant-ressource.

À l'instar des recommandations favorables de Dexter et Hugues (2011) ainsi que de Schoenbach, Greenleaf et Murphy (2012), les enseignants-ressources soutiennent que l'utilisation de l'organisateur graphique est un moyen de choix pour conduire des discussions métacognitives en salle de classe. Conformément à ce que nous avions répertorié dans les écrits, ils ont utilisé l'organisateur graphique pour faire verbaliser les élèves sur leurs connaissances antérieures, identifier les informations données dans le texte, se questionner et établir des liens entre les différentes information recueillies (Lee \& Spartley, 2010). Les propos recueillis mettent aussi en lumière le rôle essentiel de l'enseignantressource comme intervenant auprès des élèves ciblés. Il est permis de croire qu'avec davantage de formation, l'enseignant-ressource pourrait soutenir les élèves et leurs enseignants dans l'appropriation de stratégies visant à mieux lire et écrire au secondaire (Chauvin \& Theodore, 2015).

De plus, les enseignants-ressources qui se sont investis dans cette démarche collaborative ont été très bien accueillis par les élèves qui se sont montrés très réceptifs à l'égard de ce type de soutien. La formation aux caractères didactique et pédagogique enrichit à la fois la pratique de l'enseignant disciplinaire et celle de l'enseignant-ressource. Des recherches plus approfondies pourraient certainement être conduites en lien avec le développement des compétences en lecture et en écriture chez les élèves à risque ou HDAA. Puisque les enseignants-ressources vont parfois assister l'enseignant en salle de classe, il serait pertinent de s'intéresser aussi aux modalités de coenseignement (Tremblay, 2015). Ce type de pratiques ouvre sur le dialogue et entrâne un souci de cohésion au sein de l'équipe-école. Ainsi, en plus de faire le suivi des élèves ciblés, les enseignants-ressources pourront s'investir à créer des espaces d'apprentissages constructifs qui stimulent autant les élèves qu'eux-mêmes dans l'exercice de cette fonction.

\section{Conclusion}

Dans cette recherche-action, le rôle de l'enseignant-ressource était au cœur de nos préoccupations. L'utilisation de l'organisateur graphique répondait 
à un besoin formulé par les enseignants-ressources basé sur les difficultés éprouvées par les élèves à lire et comprendre des textes puis à réinvestir l'information colligée dans une tâche écrite. Le projet au sein duquel se sont engagés neuf enseignants-ressources permet de croire qu'il est possible de développer davantage les volets didactiques et pédagogiques de cette fonction. À l'instar des recommandations formulées par Kaldenberg et al. (2015), les organisateurs graphiques ont contribué à accompagner l'apprentissage et à soutenir l'appropriation de contenus disciplinaires chez des élèves en difficulté au secondaire, et ce dans différentes matières (Lee et al., 2007). Le fait que les utilisateurs verbalisent bien des fonctions de l'organisateur graphique, soit de comparer, voir, résumer, structurer, et comprendre, nous indique qu'ils se sont appropriés ces nouvelles stratégies (Graham \& Harris, 2009; Jiang et Grabe, 2007). Si certains mentionnent vouloir se les approprier davantage et tisser encore plus de liens entre la lecture et l'écriture, ces nouvelles pratiques constituent une base solide pour un accompagnement pédagogique qui tienne compte des connaissances issues de la recherche. Plus encore, nous tenions à expérimenter avec eux de nouvelles pratiques afin de mettre à l'épreuve le postulat d'éducabilité qui préconise que tous les élèves peuvent apprendre si les moyens et les stratégies mobilisés répondent à leurs besoins. L'utilisation de l'organisateur graphique a permis de constater que les ressources offertes aux élèves en difficulté doivent être davantage analysées au regard du potentiel d'apprentissage et des habilités et stratégies cognitives à développer et stimuler (Schoenbach et al., 2012). Bien que la fonction d'enseignant-ressource pourrait certainement être optimisée par une plus grande appropriation d'interventions stratégiques liées à l'apprentissage, il demeure qu'elle doive s'inscrire au sein de modèles de services fondés sur la complémentarité de ses acteurs. Il ne s'agit donc pas de faire de l'enseignant-ressource un substitut à l'orthopédagogue qui demeure un intervenant spécialisé dans la rééducation des troubles de l'apprentissage. Toutefois, l'enseignant-ressource peut aisément trouver sa place dans le quotidien de l'élève, dans la classe ordinaire ou dans des sous-groupes de besoins, au sein desquels il enseignerait explicitement des stratégies et susciterait des discussions métacognitives favorables à la construction de sens dans les disciplines scolaires.

Bien entendu, nous sommes conscientes que les résultats obtenus au cours de cette recherche exploratoire constituent davantage des indicateurs pour des recherches subséquentes. Le petit échantillon, la diversité des disciplines, niveaux scolaires et types d'organisateurs utilisés ne nous permettent pas de tirer des conclusions éprouvées. Cependant, ces résultats permettront possiblement de fournir de nouvelles pistes de pratiques dans le cadre de la fonction d'enseignant-ressource assumée par les enseignants du secondaire. Des recherches subséquentes pourront certainement s'intéresser à l'appropriation 
d'outils comme l'organisateur graphique dans un plus large échantillon de participants et documenter les apports sur la réussite des élèves à risque ou HDAA. L'idée générale de cette fonction étant de promouvoir l'utilisation d'interventions pouvant s'appliquer à tous, mais démontrant une efficience marquée chez les élèves dont on dit trop souvent qu'ils peinent à apprendre.

\section{References}

Allenbach, M., Borri-Anadon, C., Leblanc, M., Paré, M., Rebetez, F. \& Tremblay, P. (2016). Les relations de collaboration entre enseignants et intervenants en transition vers l'inclusion scolaire. Dans L. Prud'homme, H. Duchesne, P. Bonvin \& Vienneau, R. (dir.), L'inclusion scolaire: ses fondements, ses acteurs et ses pratiques (pp. 71-86). DeBoeck: Bruxelles.

Bissonnette, S., Richard, M., Gauthier, C. \& Bouchard, C. (2010). Quelles sont les stratégies d'enseignement efficaces favorisant les apprentissages fondamentaux auprès des élèves en difficulté de niveau élémentaire? Résultats d'une mégaanalyse. Revue de recherche appliquée sur l'apprentissage, 3(1).

Block, C.C. \& Pressley, M. (2007). Best practices in teaching comprehension. In L.B. Gambrell, L.M. Morrow \& M. Pressley (eds.), Best practices in literacy instruction (3rd ed., pp. 220-242). New York: Guilford.

Boudreault, P. \& Kalubi, J.-C. (2006). Animation de groupes: Une démarche réflexive d'analyse. Montréal: Éditions Carte Blanche.

Bourassa, M., Philion, R. \& Chevalier, J. (2007). L'analyse de construits: une coconstruction de groupe. Revue Éducation et francophonie, 35(2), 78-116.

Brochu, P., Deussing, M.-P., Houme, K. \& Chuy, M. (2013). À la hauteur: Résultats canadiens de l'étude PISA de l'OCDE. Le rendement des jeunes du Canada en mathématiques, en lecture et en sciences. Premiers résultats de 2012 pour les jeunes du Canada âgés de 15 ans. Toronto, On.: Conseil des ministres de l'éducation (Canada). Récupéré de: http://cmec.ca/Publications/Lists/Publications/ Attachments/318/PISA2012_CanadianReport_fr_Web.pdf.

Chauvin, R. \& Theodore, K. (2015). Teaching content-area literacy and disciplinary literacy. SEDL Insights, 3(1), spring.

Comité patronal de négociation pour les commissions scolaires francophones (CPNCF, 2011). Entente intervenue entre le Comité patronal de négociation pour les commissions scolaires francophones et la Centrale des syndicats du Québec (csq) pour le compte des syndicats d'enseignantes et d'enseignants dans le cadre de la loi sur le régime de négociation des conventions collectives dans les secteurs public et parapublic.

Récupéré de:

http://cpn.gouv.qc.ca/fileadmin/documents/CPNCF/05_Conv_coll_2010_2015/ enseignant/FSE-CSQ/2011-06-08_FSE_Conv_2010-2015_Internet.pdf.

Couture, C, Bednarz, N. \& Barry, S. (2007). Conclusion. Multiples regards sur la recherche participative, une lecture transversale. In M. Anadòn \& L. Savoie-Zajc, La recherche participative. Multiples regards (pp. 205-221). Québec: PUQ. 
Dexter, D.D. \& Hughes, C.A. (2011). Graphic organizers and students with learning disabilities: A meta-analysis. Learning Disability Quarterly, 34, 51-72.

Jiang, X. \& Grabe, W. (2007). Graphic Organizers in Reading Instruction: Research Findings and Issues. Reading in a Foreign Language, 19(1), 34-35.

Fédération des syndicats de l'enseignement (FSE) (2015-2020). Entente nationale.

Granger, N. (2012). La transformation des pratiques professionnelles chez les enseignants du secondaire: Analyse des effets de la participation aux cercles d'apprentissage et d'inclusion. Thèse de doctorat inédite: Université de Sherbrooke.

Granger, N. \& Dubé, F. (2014). Rapport de recherche programme de soutien en adaptation scolaire: MELS.

Granger, N. \& Tremblay, P. (soumis). La satisfaction des enseignants-ressources à l'égard des rôles et fonctions pour soutenir la réussite des élèves à besoins spécifiques. Enfance en difficulté.

Graham, S. \& Harris, K.R. (2009). Almost 30 Years of Writing Research: Making Sense of It All with The Wrath of Khan. Learning disabilities research and practice, 24(2), 58-68.

Graham, S. \& Perin, D. (2007). A meta-analysis of writing instruction for adolescent students. Journal of Educational Psychology, 99, 445-476.

Kaldenberg, E.R., Watt, S.J. \& Therrien, W.J. (2015). Reading Instruction in Science for Students With Learning Disabilities: A Meta-Analysis. Learning Disability Quarterly, 38(3), 160-173.

Karsenti, T. \& Savoie-Zajc, L. (2004). La recherche en éducation: étapes et approches. Sherbrooke: Éditions du CRP.

Lee, J., Grigg, W. \& Donahue, P. (2007). The nation's report card: Reading 2007 (NCES No. 2007-496). Washington, DC: National Center for Education Statistics, Institute of Education Sciences, U.S. Department of Education.

Lee, C.D. \& Spratley, A. (2010). Reading in the Disciplines: The Challenges of Adolescent Literacy. New York: Canergie Corporation of New York.

Lewis, B. \& Maas, S. (2007). QDA Miner 2.0: Mixed-Model Qualitative Data Analysis Software. Field Methods, 19, 87-108.

McCracken, M., \& Murray, T.S. (2009). National Strategy for Early Literacy. The economic benefits of literacy: Evidence and implications for public policy. London, ON: Canadian Language and Literacy Research Network.

McKenna, J.W., Shin, M. \& Ciullo, S. (2015). Evaluating reading and mathematics instruction for students with learning disabilities: A synthesis of observation research, Learning disability quarterly, 38(4), 195-2017.

Mertens, D.M. (2005). Research and evaluation in education and psychology: integrating diversity with quantitative, qualitative, and mixed methods. Thousand Oaks, CA: Sage Publications.

Ministère de l'Éducation, des Loisirs et du Sport (2007). L'organisation des services éducatifs aux élèves à risque et aux élèves handicapés ou en difficulté d'adaptation ou d'apprentissage. Récupéré de: http://www.education.gouv.qc.ca/fileadmin/site_ web/documents/dpse/adaptation_serv_compl/19-7065.pdf.

Ministère de l'Éducation, des Loisirs et du Sport (2008). Évaluation de programme: Plan d'action sur la lecture. Récupéré de: http://www.mels.gouv.qc.ca/sections/ publications/index.asp?page $=$ fiche $\& i d=820$. 
National Center for Education Statistics (2009). The nation's report card: Reading 2009. Washington, DC: National Center for Education Statistics, Institute of Education Sciences, U.S. Department of Education.

OECD (2010), PISA 2009 Results: What Students Know and Can Do - Student Performance in Reading, Mathematics and Science (Volume I). Récupéré de: http:// dx.doi.org/10.1787/9789264091450-en.

Porter, G. \& Aucoin A. (2012). Consolider l'inclusion pour consolider nos écoles: Rapport du processus de révision de l'inclusion scolaire des écoles du NouveauBrunswick. Présenté au ministre de l'Éducation et du Développement de la petite enfance; N-B.

Prud'homme, L., Dolbec, A. \& Guay, M.-H. (2011). Le sens construit autour de la différenciation. Éducation et francophonie, 39(2), 165-188.

Rouhani, Y., Moulavi Nafchi, A. \& Mehdi Ziaee, S. (2016). Applying Different Interventions to Teach Writing to Students with Disabilities: A Review Study. Theory and Practice in Language Studies, 6(4), 733-741.

Scammacca, N.K., Roberts, G., Vaughn, S. \& Stuebing, K. (2015). A Meta-Analysis of Interventions for Struggling Readers in Grades 4-12: 1980-2011. Journal of Learning Disabilities, 48(4) 369-390.

Schoenbach, R., Greenleaf, C. \& Murphy, L. (2012). Reading for understanding: How Reading Apprenticeship improves disciplinary learning in secondary and college classrooms. San Francisco: Jossey-Bass.

Shanahan, T. \& Shanahan, C. (2008). Teaching disciplinary literacy to adolescents: Rethinking content-area literacy. Harvard Educational Review, 78(1), 40-59.

Strangman, S., Hall, T. \& Meyer, A. (2003). Graphic organizers and implications for universal design for learning: Curriculum enhancement report, National Center on Accessing the Genaral Curriculum.

Thomazet, S. (2008). L'intégration a des limites, pas l'école inclusive! Revue des sciences de l'éducation, 34(1), 123-139.

Tremblay, P. (2015). Le coenseignement: condition suffissante de différenciation pédagogique? Formation et profession, 23(3), 33-44. 